\title{
Applications of Brain-Machine Interface Systems in Stroke Recovery and Rehabilitation
}

\author{
Anusha Venkatakrishnan • Gerard E. Francisco • \\ Jose L. Contreras-Vidal
}

Published online: 22 April 2014

(C) Springer Science + Business Media New York 2014

\begin{abstract}
Stroke is a leading cause of disability, significantly impacting the quality of life (QOL) in survivors, and rehabilitation remains the mainstay of treatment in these patients. Recent engineering and technological advances such as brain-machine interfaces (BMI) and robotic rehabilitative devices are promising to enhance stroke neurorehabilitation, to accelerate functional recovery and improve QOL. This review discusses the recent applications of BMI and robotic-assisted rehabilitation in stroke patients. We present the framework for integrated BMI and robotic-assisted therapies, and discuss their potential therapeutic, assistive and diagnostic functions in stroke rehabilitation. Finally, we conclude with an outlook on the potential challenges and future directions of these neurotechnologies, and their impact on clinical rehabilitation.
\end{abstract}

Keywords Brain-machine interfaces - Robotic-assisted rehabilitation - Robotic exoskeletons - Stroke .

Neurorehabilitation · Functional recovery ·

Neuroplasticity $\cdot$ Clinical trials

A. Venkatakrishnan $(\bowtie) \cdot$ J. L. Contreras-Vidal

Laboratory for Non-invasive Brain-Machine Interface Systems,

Department of Electrical and Computer Engineering, University

of Houston, Houston, TX, USA

e-mail: avenkatakrishnan@uh.edu

G. E. Francisco

Department of Physical Medicine and Rehabilitation, University of Texas Health Science Center, Houston, TX, USA

G. E. Francisco

NeuroRecovery Research Center, TIRR Memorial Hermann

Houston, Houston, TX, USA

\section{Introduction}

Cerebrovascular diseases, or strokes, affect approximately 795,000 people every year in the United States alone, and according to the Survey of Income and Program Participation (SIPP, a survey of the US Bureau of the Census), stroke is a leading cause of serious, long-term disability [1]. With at least $50 \%$ of survivors experiencing some hemiparesis, it accounts for the poor physical health and the social dysfunction evident in survivors [2]. A 2005 Centers for Disease Control and Prevention (CDC) survey indicated that only $30.7 \%$ of stroke survivors received outpatient rehabilitation, which was much lower than what would be expected if clinical practice guideline recommendations had been followed for all stroke patients. Therefore, increasing access to neurorehabilitation would consequently increase functional recovery and long-term quality of life (QOL) in these patients, while allowing them greater participation in society. Lack of functional independence to access outpatient facilities, and more importantly, rehabilitation costs and reimbursement caps, can be significant rate-limiters in functional recovery and enhancing independent quality of life. Harnessing recent advances in brain-machine interfaces (BMI) and robotic-assisted rehabilitation technologies has the potential not only to promote functional restitution through sensorimotor adaptation and central nervous system plasticity [3], but also help reduce the socio-economic burden of disability $[4,5]$. By adjusting parameters tailored to each individual, his/her state of disability, and goals of intervention, these technologies can provide greater durations of consistent, patient-engaged, repetitive motor practice that consequently allow a physical therapist to work with more patients in the same allotted time. Moreover, BMIs can also be used as a method to measure functional recovery and neuronal plastic changes $[6-8,9 \bullet \bullet]$. This review provides an 
overview of BMIs and robotic devices, and discusses how the integration of these two technologies may significantly enhance clinical stroke rehabilitation and understanding of brain function. Further, the challenges in translating these research technologies to the clinic are also presented, with future directions for this field.

\section{Brain Machine Interface (BMI) Technologies}

BMI systems infer the user's intent from neural data acquired from the brain, and transform it into output variables to control screen cursors, prosthetic devices, assistive orthotic devices, etc., in real time. One of the first implementations of a brain-computer interface consisted of using an event-related potential (ERP) associated with the classical oddball paradigm to identify letters in the alphabet, which helped the user communicate through words [10]. Since then, the school of thought that primarily considered neural interfaces to be applicable only in the completely paralyzed and/or individuals who are "locked-in" and cannot communicate verbally has definitively changed, and BMIs are getting integrated into mainstream rehabilitation. The reasons for this are primarily: (a) ability to measure brain signals non-invasively that can be effectively transformed into control signals, using methods such as electroencephalography (EEG) [11, 12], magnetoencephalography (MEG) [13], and functional near-infrared spectroscopy (fNIRS) [14, 15]; (b) improvements in technology that allow relatively fast analysis of large-scale, multidimensional data sets; and (c) increased understanding of neuroplastic mechanisms of motor learning and adaptation [16, 17] and functional motor recovery [18], which has further catalyzed use of brain-derived neural signals in rehabilitative BMIs.

BMIs have the potential to greatly improve clinical rehabilitation regimens by using extant neurological signals to drive and enhance functional recovery by actively engaging the user in rehabilitation, while simultaneously allowing for monitoring and quantification of internal states and neural plasticity over time. Also, the process of learning to use the BMI, i.e., learning to control the device/perform the task at hand using neural "thought" signals is a process of cognitivemotor learning, which is of benefit in recruiting existing neuroplastic mechanisms. Besides, successful task performance is fed back in the loop, recruiting error-correcting and reward-related feedback mechanisms. Taken together, these recent BMI applications for traininginduced plasticity have made it an important rehabilitative tool, rather than a mere substitutive tool for the severely impaired patients.

\section{Clinician Benefits with Brain Machine Interfaces}

Motivation is an important psychosocial factor that can greatly affect neurological rehabilitation outcomes [19]. Therefore, active user engagement and positive reinforcement provided through both neural signals as well as task goal accomplishment, e.g., using an upper limb orthotic device that allows a stroke patient to move a paretic arm to grasp an object, a task that can otherwise not be performed by the patient, can significantly enhance patient motivation. The impact of this on enhancing rehabilitation outcomes could be profound, and is generally underscored. Secondly, BMIs can allow physical therapists and other rehabilitation clinicians to have continuous access to neural monitoring during treatment. This allows for personalizing treatment to each individual based on his/her functional abilities at a level of granularity that is otherwise impossible. Most importantly, these neural markers can be used to guide changes in treatment parameters, i.e., increasing/decreasing task difficulty or challenges, as well as allowing for task modifications. In other words, neural data can be used for neurological rehabilitation in the same manner as VO2 max or electrocardiogram (EKG) is used for cardiac rehabilitation, i.e., as a window into the internal physiological state that informs the clinician to appropriately modify exercise levels. This can significantly help clinicians and patients alike by helping make treatment protocols personally adaptive, as well as minimizing injury due to fatigue.

\section{Brain-Machine Interfaces in Stroke Rehabilitation}

The use of BMIs in stroke neurorehabilitation has become popular in recent times, given their benefits of guiding and enhancing neuromotor learning. Neural control signals may be obtained for a BMI via implanted electrode arrays (including electrocorticography, i.e., ECoG) or through techniques that measure neural activity on the scalp directly (e.g., EEG and MEG) or indirectly (e.g., blood oxygenation levels through functional magnetic resonance imaging i.e., fMRI and fNIRS). For the purpose of this review, BMI techniques employing measurements of scalp neural activity are discussed, as this is non-invasive and more relevant to stroke rehabilitation.

In this context, mu-rhythm, i.e., $8-13 \mathrm{~Hz}$ oscillatory activity observed over the central sensory-motor scalp areas in EEG and MEG, has been found to be quite successful as a neural control signal for BMIs [13, 20, 21••, 22]. Eventrelated desynchronization (ERD) or reduction in amplitude of this oscillatory band activity in response to a stimulus/Go cue has been used to control the impaired upper limb orthotic devices with some success in stroke patients. Patients improved in achieving task successes over multiple training sessions [13], which further substantiated the notion that 
BMIs can recruit extant neuroplasticity in chronic stroke patients. More recently, a larger-scale controlled clinical study demonstrated that stroke patients with minimal hand function who received ERD-driven BMI training as an adjunct to physical therapy to control a hand-orthotic device showed functional improvements in Fugl-Meyer assessment scores, compared to those who received sham BMI training (non-neural control of orthosis) [21]. Furthermore, the functional improvements in these patients were also significantly correlated with hand electromyographic activity, thereby providing evidence of peripheral neuromuscular plasticity driven by BMI training. These findings provide great promise for the future of BMI use in clinical stroke rehabilitation. Further, since motor imagery is used by patients with paresis or paralysis in order to generate neural signals simulating movement in the brain, this provides an additional avenue to engage neuroplastic mechanisms in stroke patients [23].

BMIs can also be coupled with functional electrical stimulation (FES) in order to allow more intentional control of FES of relevant muscles. It is postulated that neurally driven FES can engage Hebbian mechanisms of associative learning and consequently increase synaptic plasticity. A recent study [24] has shown the feasibility of using mu-rhythm ERD to drive FES of the tibialis anterior (TA) in a stroke patient. Interestingly, the authors found increased EMG activity in the TA, along with increased dorsiflexion, following BMI-FES rather than FES alone. This is very promising, as improving TA muscle control and dorsiflexion range of motion (ROM) can significantly impact gait training in stroke patients and improve functional recovery.

Recently, BMI coupled with virtual reality (VR) environments have also gained popularity in the context of stroke rehabilitation. Virtual environments have been very useful to train functional upper limb pointing movements in stroke patients [25, 26, 27]. Therefore, adding a neural interface to VR training can help engage patients early on in the stages of functional recovery when volitional movement may be more limited. The benefits would include increased recruitment of cortical motor networks through motor imagery used to control the BMI, as well as engaging motor learning mechanisms through repetitive training. Researchers have developed and tested a prototypical VR system in healthy individuals that involves controlling a virtual 'avatar' using a motor-imagery based BMI [28]. The use of such BMIbased VR rehabilitation in early stages of stroke recovery could significantly alter the trajectory of functional recovery in patients, thereby enhancing quality of life and potentially reducing needs for long-term rehabilitation and associated costs.

\section{Robotic Rehabilitation Devices}

Neurological rehabilitation in stroke survivors is primarily focused on harnessing neural plasticity of the central nervous system to restore functional mobility in terms of normal, energy-efficient movement patterns. This is achieved through repetitive, task-oriented and goal-oriented motor practice in direct interaction with the physical therapist [19]. By definition, this rehabilitation is "adaptive" to patient needs, thereby requiring constant adjustments to the treatment regimen in terms of both type of exercise and dosage of exercise. However, as with any form of motor learning, repetition or practice remains a central tenet of neurological rehabilitation [29]. This principle allows for integration of robotic devices into rehabilitation, since these can be programmed to provide repetitive, task-oriented practice in an objective and consistent manner.

A brief overview of current upper limb and lower limb robotic devices and their applications in stroke rehabilitation is presented in this section. Typically, rehabilitation robotic devices are divided in two categories: therapeutic use systems and personal use systems [30]. Therapeutic systems are primarily designed for use in the clinical setting, and are used across multiple patients/users, while personal systems serve as assistive devices for a single patient/user in their home environment to aid completion of activities of daily living (ADL). Further, therapeutic robotic systems may be classified into active and passive systems. Active systems have motorized actuators to simulate a joint and can produce movement in that given degree of freedom (DOF). On the contrary, passive systems simply allow for movement of the upper or lower limb segment through the given DOF wherein muscle activity of the user generates torque for movement; this can aid in movement diagnostics. For the scope of this review, therapeutic systems will be discussed, as these are pertinent to the current discussion of BMI-based robotic rehabilitation.

\section{Upper Limb Robotic Devices}

The MIT-Manus robot, developed at the Massachusetts Institute of Technology (MIT) in the early 1990s, was the first robotic device designed specifically for upper limb rehabilitation [30, 31]. This is an active robot that allows for two DOF motions, i.e., at the shoulder and elbow to perform anti-gravity movements. Forces and movement are transmitted to the user's hand through a gripped robotic manipulandum [30]. The manipulandum has low inertia and the motors are also back-drivable, such that the device can be operated in a passive mode. Therefore, the device can be used with variable assistance from complete robot- 
driven forces to completely user-driven movement. More recently, the MIT-Manus also can be equipped with an additional wrist unit for flexion-extension, abductionadduction, and forearm pronation-supination movements; and a grasp-hand unit for closing and opening movements [31]. The largest multi-site clinical study examining the effectiveness of the MIT-Manus (along with the wrist-hand attachment) in upper limb rehabilitation in chronic stroke patients found that robotic-assisted therapy improved functional clinical outcomes (Fugl-Meyer assessment scores) compared to usual therapy, but not with respect to intensive comparison therapy at 36-week follow-up [32]. Though improvements in comparison to usual therapy were modest, this study was the first to demonstrate functional improvements in a heterogeneous group of chronic stroke survivors, thereby providing evidence for neural plasticity in these patients. Additionally, this evidence also substantiates the need to standardize upper limb rehabilitation protocols across clinics and hospitals, and robotic devices allow for a more seamless standardization process.

Another type of upper limb robot is one that is modeled as an exoskeleton that can be interfaced with the upper limb rather than as a robotic manipulandum, namely the ARMin robotic semi-exoskeleton [33, 34]. This device has six DOF, and has both position and force sensors. The distal part, characterized by an exoskeleton, moves only the elbow, whereas the shoulder joint is actuated by an endeffector part connecting the upper arm with the wallmounted axes, allowing for vertical as well as horizontal shoulder rotation (i.e., flexion/extension and abduction/ adduction in both planes). Additionally, a special custommade upper arm rotary module connected to the upper arm via an orthotic shell achieves shoulder internal/external rotation. This three-dimensional shoulder movement allows to simulate ADL by coupling proximal shoulder and distal elbow movements, and can help train functional multi-joint synergistic movements. A recent study combined the ARMin with a hand grasp robotic device (HANDSome) and found that upper limb training for $12 \mathrm{~h}$ over 3 months with this combined robotic device improved function in stroke patients as compared to conventional therapy [35••]. This promising finding from a pilot study of 12 patients suggests that these technologies could have a real and positive impact on improving rehabilitation outcomes.

An issue with using exoskeletal devices is that if appropriate alignment between the physiological joints and robotic actuators or "joints" is not achieved, it can tend to create excessive forces across limb segments and/or joints, and potentially cause damage. This is an important focus for current research efforts, to optimize design to ensure appropriate and near-perfect alignment of the robotic device with the user. In this regard, the NEUROExos, is a new upper limb exoskeleton that has four DOF with a functionally actuated elbow designed to be used for stroke rehabilitation [36]. Importantly, this device has a compact and lightweight mechanical structure with double-shelled links, and a wide physical human-robotic interaction surface area to minimize the pressure on the skin. This makes the device much more user-compatible, and is an important design advancement. This device allows for elbow rotation in the frontal plane, elbow rotation in the horizontal plane, translation of the forearm link along the flexion-extension axis, and translation in the horizontal plane. Within these DOFs, an antagonistic, compliant, remote actuation system exists with an independent joint position and stiffness control (for robot-in-charge exercises) and near-zero impedance torque control (for patient-in-charge exercises). This allows for adjusting the treatment protocol based on the user's functional status, thereby optimizing therapeutic efficacy of training. Additionally, using a compliant actuator control system with forces within normal physiological ranges prevents harmful effects that could arise when the robotic device interacts with an excessively spastic arm.

In terms of focusing on distal upper limb segments, the MAHI EXO-II (based on the RiceWrist) is a flexible five DOF, electrically actuated upper-extremity haptic exoskeleton device [8, 37, 38]. This device allows for three therapeutic modes, to tailor treatment to the subject's motor abilities: passive, triggered, and active-constrained. In the passive mode, the robot performs the movement. In the triggered mode, the subject has to overcome a threshold resistance force before the robot takes over and completes the movement. In the active-constrained mode, the subject must execute movements against resistance. Preliminary studies in our partner laboratories have shown promising findings using this device for wrist-hand function stroke patients. Currently, a clinical study with a larger sample of stroke patients is underway (ClinicalTrials.gov, NCT01948739).

Another robotic device primarily designed to train hand movements is the Hand Wrist Assistive Rehabilitation Device ('HWARD'), a three DOF, pneumatically actuated device that assists the hand in grasp and in release movements [39]. This device allows flexion/extension of the four fingers together about the metacarpophalangeal (MCP) joint, flexion/extension of the thumb at the MCP joint and flexion/extension of the wrist. In this study, 13 chronic stroke patients were trained for 15 daily sessions over 3 weeks using the HWARD device, wherein subjects were given feedback about the robot active-assisted hand movements on a computer monitor (in an augmented VR setting). Interestingly, it was found that patients showed significant functional gains compared to pre-training levels (in Action Research Arm Test and Fugl-Meyer 
Assessment). Importantly, these functional gains were correlated with cortical reorganization maps as seen in fMRI; this provided a critical piece of evidence for neuroplasticity modulated by robotic-aided rehabilitation.

The aforementioned discussion is only a brief review of upper limb robotic rehabilitation devices, as applicable in the context of interfacing with BMI. For detailed reviews, readers are referred to [30, 40, 41, 42•, 43]. Nevertheless, early findings using these robotic devices highlight the promise for using this technology in mainstream stroke neurorehabilitation.

\section{Lower Limb Powered Robotic Devices}

Gait training is an important goal in stroke rehabilitation to enable functionally independent ambulation in these patients. Design of lower limb powered robotic devices faces the additional challenge of accounting for body weight support, some way of achieving balance control, and transfer of weight between limbs necessary for normal gait. In the context of lower limb robotic devices, treadmill-based robotic gait training devices were among the first to be designed, namely the Lokomat ${ }^{\mathrm{TM}}{ }^{\circledR}$ (Hocoma) [44] and the Lower Extremity Powered Exoskeleton $\left(\mathrm{LOPES}^{\mathrm{TM}}\right)$ [45]. These devices allow for gait training on a treadmill with actuated DOF for the lower limb joints with variable body weight support. They primarily focus on promoting more normal gait patterns via the robotic actuators repetitively guiding both the paretic and unaffected lower limb segments through pre-programmed gait cycles. The main advantage with such training is that the stroke patient can practice functional multi-joint synergistic movement patterns, which can improve motor recovery through motor learning.

There are, however, some important differences

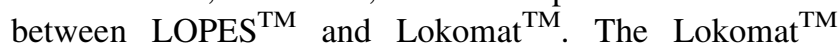
device has four DOF (bilateral hip and knee flexion/ extension) that are powered (actuated) by position-controllers. This means that the multi-joint movement patterns in the Lokomat ${ }^{\mathrm{TM}}$ device are primarily pre-programmed and fixed. The patient is expected to adapt to the device's walking pattern, and thereby re-learn normal gait patterns. In a stroke patient, the control algorithm was adapted (to control both position and force interactions via an impedance controller) so that the affected leg's movement patterns are programmed to be phase-shifted (180 degrees, i.e., anti-phase) with respect to the normal leg movements that are allowed more freely [44]. This should encourage alternating, repetitive and symmetric movement patterns in both legs, which will consequently improve gait asymmetries in stroke patients. Also, biofeedback about the user's muscle activity can be provided while training on the Lokomat $^{\mathrm{TM}}$, which can help engage the user as well as serve as a clinical marker for functional improvements [46]. On the other hand, the LOPES ${ }^{\mathrm{TM}}$ has eight DOFs, namely, pelvic left/right movement, forward/backward movement, hip flexion/extension and abduction/adduction and finally knee flexion/extension. Ankle movements are not actuated, and are allowed to freely move. Additionally, in the LOPES ${ }^{\mathrm{TM}}$, movements are controlled based on low impedance control principles, such that both position and force interactions at the joint (or DOF) are controlled [47]. The LOPES ${ }^{\mathrm{TM}}$ gait trainer is also modeled more on an exoskeleton principle, thereby requiring appropriate alignment of physiological and robotic joints. The impedance controller algorithm allows for a spectrum of training modes ranging from those when the robot is fully in charge training the patient in pre-programmed gait movement patterns, to when the therapist is in charge, where the actuators serve as force sources acting upon previously decided force patterns, to finally, the patient in charge, with the patient having control over movement patterns. These allow for progressive variations in treatment schedule. Both the Lokomat ${ }^{\mathrm{TM}}$ and LOPES $^{\mathrm{TM}}$ devices can be augmented by ankle orthotic devices or powered ankle actuators, such as the AnkleBOT ${ }^{\mathrm{TM}}$ or pneumatic powered Ankle-foot orthosis [48], in order to functionally train ankle movement during gait [49]. In a multi-center clinical trial [50], it was found that robotic training with the Lokomat $^{\mathrm{TM}}$ did not improve functional gait patterns in subacute stroke survivors compared to conventional gait training. The authors concluded that this was likely due to the lack of diversity in the robotic training protocol. However, in a study wherein acute stroke survivors were trained with the Lokomat ${ }^{\mathrm{TM}}$, it was found that these patients showed much higher functional gains in terms of ambulation scores and NIH Stroke scale scores (NIHSS) as compared to conventional physical therapy [51]. This raises an interesting issue about the need for identifying optimal treatment protocols based on the time for intervention, i.e., in the acute, sub-acute or chronic stages of stroke recovery. Recently [52], it was found that using a modified control algorithm incorporating a virtual ankle trajectory, based on end-point control rather than absolute joint control, in the LOPES ${ }^{\mathrm{TM}}$ trainer actually improved gait patterns in stroke patients. While this was not a longitudinal study investigating functional gains over time, it definitely provides evidence to substantiate the fact that robotic devices can greatly enhance clinical rehabilitation protocols when used to train focused, functional movement patterns appropriately. It is expected that large-scale clinical studies examining efficacy of these devices employing novel control algorithms should soon help determine the utility of these devices in clinical stroke rehabilitation.

The Active Leg Exoskeleton (ALEX) [53] is a robotic exoskeleton-based device with seven DOF: three at the 
trunk, i.e., vertical and lateral translations and rotation about a vertical axis; two for movements of the thigh segment, i.e., flexion/extension and abduction/adduction; one for movement of the shank segment, i.e., flexion/ extension (of knee); and finally, one for movement of the foot, i.e., ankle plantarflexion/dorsiflexion. A walker device is attached to and supports the weight of the device. This device operates based on a force-field controller by applying tangential and perpendicular forces at the ankle. The tangential forces help move the ankle of the patient along the trajectory, and perpendicular forces generate simulations of virtual-walls around the desired ankle trajectory in the plane containing the human thigh and shank, which the patient has to overcome to move along as occurs in gait. This force-field controller helps in rehabilitation by acting in an active assistive mode, wherein the tangential forces provide assistance to overcome the perpendicular resistive forces along the sagittal plane of the lower limb. The tangential force can act as proprioceptive feedback, and can be decreased as the patient improves. In a pilot study [53], two stroke patients were trained using the ALEX employing the force-field controller, and also provided visual feedback of the desired ankle path for 15 sessions. Interestingly, it was found that the patients showed improved walking speeds (on the treadmill), as well as improved ankle trajectories that resembled those of healthy controls and increased joint movements of the affected leg in the swing phase. This provides additional support for the fact that robotic-assisted gait training can be helpful, given optimal training parameters and control algorithms.

The GAIT-ESBIRRO device is designed on the principle of a wearable exoskeletal system, and is the first of its kind with bilateral hip, knee and ankle actuators [54, 55]. This exoskeleton is modeled similar to a bilateral hip-kneeankle-foot orthosis, except that it has powered joints/ actuators, which can move lower limb segments through desired trajectories. This device is particularly novel in its modular design, thereby allowing use of various segments/ modules as necessitated by a given patient's functional needs, and tailoring a treatment protocol specific to that patient. Additionally, the device is also equipped with interaction torque sensors, which can provide crucial information about interaction between patient-generated and robot-generated torques, and help monitor functional gains achieved during the course of training. One limitation of this device is the lack of body weight and exoskeletal weight support incorporated into the device. However, this allows for the device to be significantly lighter than its counterparts, as expected. Additionally, as with other exoskeletons, the physiological and robotic joint interfaces must be correctly aligned to prevent any excessive/harmful forces to be generated on the lower limb. The GAIT-
ESBIRRO exoskeleton employs an intermittent joint control strategy wherein physiological joint rigidity is selectively controlled by modifying exoskeletal joint stiffness through torques generated by external control algorithms. This mode also allows for active-assisted rehabilitation protocols, which is critical for stroke rehabilitation, and simultaneously allows monitoring patient generated interaction torques. A collaborative effort between our research team and the Spanish National Research Council (CSIC) team in Madrid, Spain is currently underway to test the efficacy of lower limb training with the recent version of the GAIT, namely the $\mathrm{H} 2$ powered exoskeleton [56], in improving gait in stroke patients.

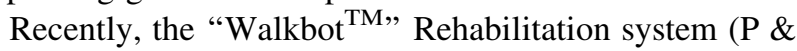
S Mechanics, South Korea) has been developed, which combines a powered lower limb exoskeleton with a treadmill, along with protective harnesses [57]. This integrated system also provides visual feedback to the patient about movement trajectories, which can improve patient engagement and motivation. Clinical investigations of efficacy using this system are not yet available to report. Further, the new Walking Assist Device (Honda R \& D Co., Ltd) is currently the most compact, commercially available, powered robotic lower limb device. The hip joint motors are activated (control algorithm) based on information obtained from hip angle sensors while walking, to provide active assistance in hip flexion/extension. This can help improve symmetry in gait swing phase bilaterally, and promote a longer stride for easier walking. Currently, one study examining clinical efficacy of this device in stroke patients is underway (ClinicalTrials.gov, NCT01994395).

Finally, this section will conclude with the discussion of the X1 Exoskeleton, a 10-DOF wearable robotic device created through a partnership between NASA (Johnson Space Center) and the Florida Institute for Human and Machine Cognition (IHMC). In this device, four DOF are actuated (knee flexion/extension and hip flexion/extension for each leg; all in the sagittal plane) and six DOF are passive (hip internal/external rotation, hip abduction/ adduction, and ankle plantarflexion/dorsiflexion for each leg). The passive DOF can be mechanically locked in position, depending on the level of active control of trunk and lower limbs in a given user. Control modes of the X1 include a dynamic position trajectory generator, which commands hip and knee joint angles to replicate a desired gait, based on inputs such as step height, step duration, and step length. Alternatively, in a user-in-charge "passive" control mode, the motors respond to any knee or hip movement to "match" the user's desired joint angle. Although this device was initially designed for astronaut exercising applications, given its versatility in terms of allowing over ground walking, we are currently investigating the feasibility of using this device in gait rehabilitation 
for hemiparetic stroke patients at the TIRR Memorial Hermann Hospital and the University of Houston.

To summarize, it is evident that robotic-assisted gait training (RAGT) is proving to be an important tool for clinicians to improve functional ambulation in stroke patients. However, important questions still remain about the determination and development of optimal control modes or strategies that can best interface with the user at each stage of recovery and maximize training benefits. For detailed reviews of lower limb robotic devices applicable to other populations in addition to stroke survivors, readers are referred to $[5,55,58]$.

\section{Combining BMI and Robotic-Assisted Rehabilitation: Challenges and Future Directions}

Given the advantages of BMI and robotic-assisted devices in stroke rehabilitation, a natural step is to combine these two technologies to integrate into mainstream rehabilitation $[59,60 \bullet, 61]$. Together, these technologies can ensure patient engagement and empower the patient with an active role in regaining function and wellbeing. In this regard, there have been successful approaches to combine BMIs with upper limb robotic devices in stroke patients [13, 21••, $62,64]$. Compared to the hand orthosis used in the studies by Buck et al. [13] and Ramos-Murguialday et al. [21••], the MIT-Manus was used as the robotic device to be controlled by a motor-imagery based BMI in stroke patients in the studies by Ang et al. [62, 63]. On the other hand, Gomez-Rodriguez et al. [64] used a Barrett robotic arm as the device that patients controlled using an EEGbased BMI. To the best of our knowledge, there have been no studies yet documenting combined BMI systems with lower limb robotic devices in the context of stroke rehabilitation. Further, the study by Ramos-Murguialday et al. [21••] is the only one that examined functional gains associated with BMI-combined robotic-assisted training over a longitudinal period of time (as discussed earlier). Therefore, it is clear that combined BMI-robotic training for stroke rehabilitation is still in its early stage of translation as a clinical therapeutic modality. There are definitely certain important issues to be considered in making this leap from the lab to the clinic using this technology; this discussion will briefly address some of these challenges and the future directions for applying this technology in clinical rehabilitation.

\section{Shared Control}

The use of higher order features in the neural signals provides additional control strategies for the patient. Shared control allows for optimal and adaptive interaction between the patient's BMI control and the pre-programmed robotic-control of the device, such that contextual information about the patient and task at hand is more seamlessly integrated into the control algorithms [59]. This will greatly improve efficiency of task performance, and therefore training can be translated to functional task settings. This is an important step to accelerate functional recovery using combined BMI-robotic assisted therapy, because clinical rehabilitation requires adaptive training of functional tasks in real-life contexts or settings [65].

\section{Adaptation}

"Adaptation" within the user-BMI-robotic device loop is important. It allows for changes within the algorithm that is extracting and optimizing the BMI control signal such that it is in line with the user's capabilities as they change over the course of training. This is critical, because as patients improve with training, it is expected that the spatial and temporal distribution of their neural activity will change indicative of neuroplasticity triggered by the training itself. If so, in the absence of an adaptive algorithm, the performance levels will tend to decrease or be inconsistent with the improved neural capacity of the patient. This will be detrimental to rehabilitation. In conventional physical rehabilitation, task demands and difficulty are progressively increased to challenge an improving patient. Similarly, by adapting itself to changing neural activity patterns, the algorithm can continue to keep up with patient needs and therefore help the patient progress favorably on the continuum of functional recovery. Alternatively, within any given session, if the patient is not performing well due to any reason such as fatigue, lack of attention to the task, etc., the adaptive algorithm can consequently be modified to reduce task demands in order to prevent the patient from getting frustrated and demotivated by poor performance on difficult tasks.

\section{Hybrid BMI}

As the name suggests, the hybrid BMI can be programmed to use additional physiological signals as inputs in addition to one or more brain-based neural signal [59]. These can include electromyography activity, heart rate, other functional neuromodulation tools such as non-invasive brain stimulation, etc. The advantage with these hybrid BMIs is that when various physiological signals are coupled, the reliability of intention detection, and consequently robustness of the control algorithm, can be significantly increased. Additionally, these various signals can also be programmed to control different aspects of the robotic device, e.g., different DOFs. Finally, the combination of these different signals can also be more useful to create an 


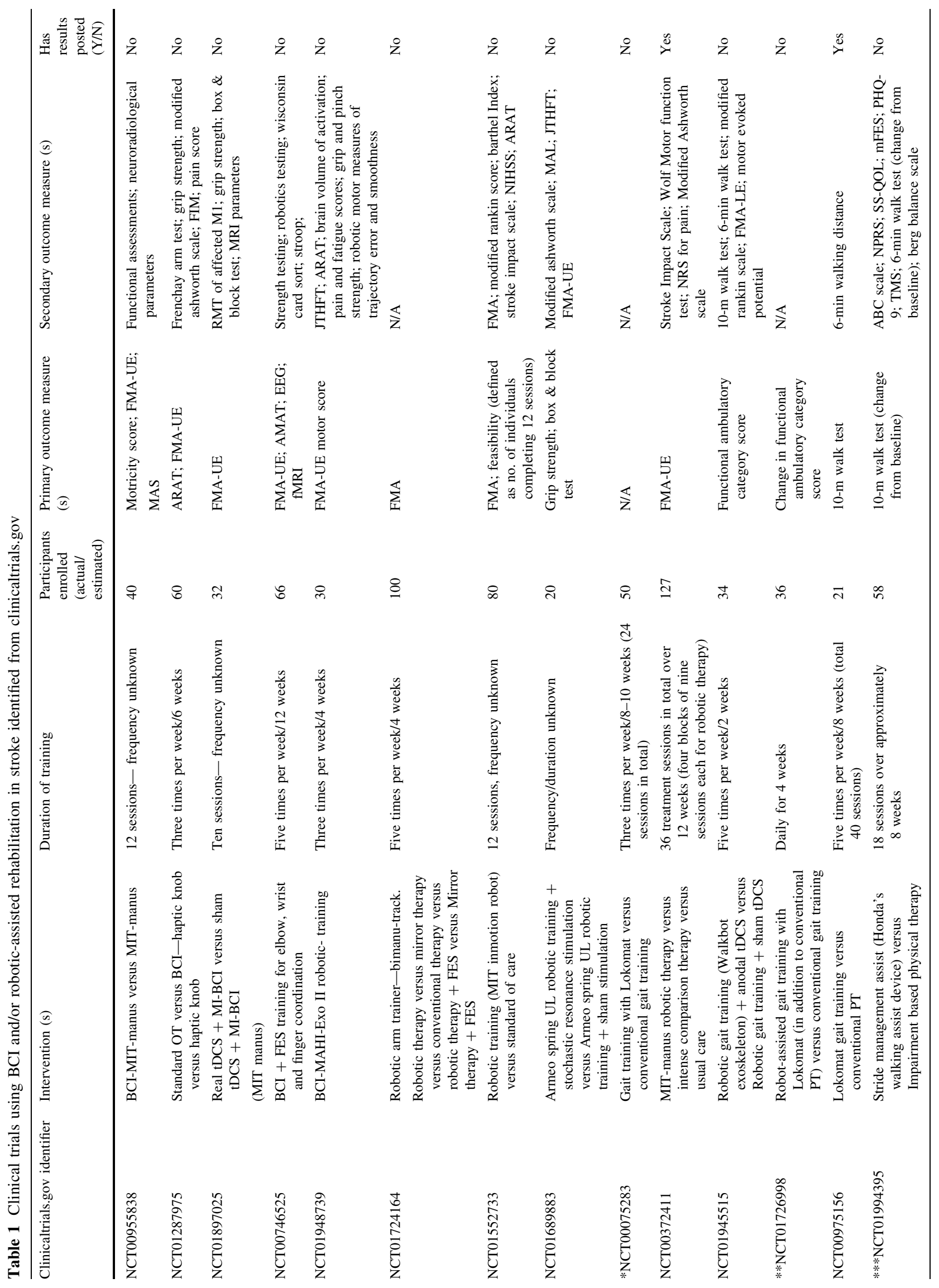


"adaptive" BMI because additional features can be used to create a more complete construct of the task context and needs, along with the patient's internal state.

\section{Clinical Outcome Metrics}

The aforementioned discussion leads to the obvious question: What physiological and/or biomechanical markers or outcomes should be used to monitor patient performance? Selection of optimal outcome variables is critical not only to assess cognitive-motor performance, but also to directly help drive shared control as well as adaptation based on current performance. This is presently an important challenge area in integrated BMI-robotic assisted rehabilitation, and it is also reflected in the variability of primary and secondary outcomes reported in ongoing clinical trials (see Table 1). Efficacy of conventional physical therapy is tracked based on functional evaluations and other clinical assessment tools for motor performance. While these are ecologically valid and assessed in real-life settings, it is unclear how robotic-assisted therapy could directly influence them. Task-specific training is an important principle in rehabilitation, and since settings for robotic-training fundamentally differ from those in which these clinical evaluations are performed, it is possible that these tests may not always be sensitive to capture the functional changes mediated by BMI-based robotic rehabilitation. Further, the sensitivity of clinical scales to functional changes also tends to be over a longer training duration. Thus, in order to track session-to-session changes in performance, it is becoming more and more evident that novel functional outcomes must be generated and quantified within single sessions as well (see Fig. 1). This is becoming an important issue in development of integrated BMI-robotic rehabilitation therapies. Given the amount of physiological measurements that occur within a BMIrobotic assisted therapy session, namely neural signals, kinematics and kinetics of task performance, it is conceivable that we should soon have composite measures of functional performance. Importantly, these metrics can be provided as real-time feedback to the patient in this human-robot interaction setup, thereby further helping to guide better performance. Further, since these novel outcome measures will be developed in the context of BMIrobotic assisted motor performance, they can be tuned to be sensitive to the subtle changes occurring as a result of motor learning. This will help provide the clinician with more direct and prompt access to the patient's current internal state, and therefore enable treatment protocol changes that are specifically tailored to the patient's needs in the timeliest manner. Additionally, these can also lead to development of novel markers of neuroplasticity that can help identify a patient's functional capabilities, i.e., serve 
Fig. 1 Summary of user within the neural-robotic interface loop. Combined BCI-robotic assisted rehabilitation, in addition to therapy, provides multimodal physiological data characterizing movements within each training session. Informed data mining can help generate reliable, robust and sensitive "biomarkers" and/or outcomes of neuromotor plasticity and recovery, which in turn will be enable the clinician to track patient progress, as well as make directed adjustments to treatment parameters. Delivery of such tailored treatments can help accelerate functional recovery in stroke survivors

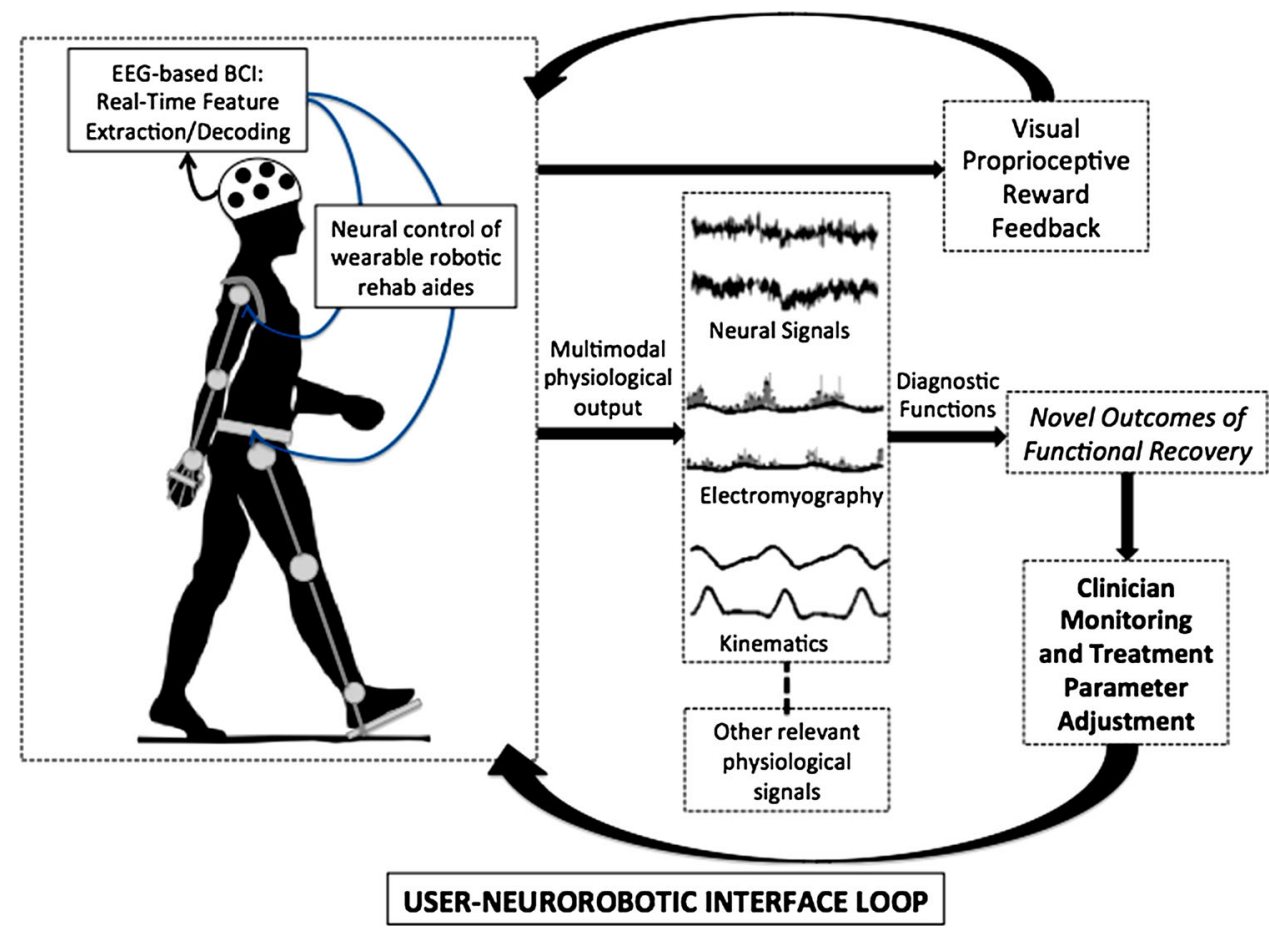

as a diagnostic marker. Therefore, integrated BMI-robotic assisted technologies are promising to be excellent diagnostic tools in addition to assistive and therapeutic tools.

\section{Future Directions}

Future directions in BMI/BMI-robotic assisted rehabilitation research should include careful, controlled clinical investigations of reliability, safety and efficacy. Specifically, multicenter clinical trials will help provide more information about the potential to use these technologies in various types of clinical settings. Given the subjectivity and variability in conventional therapy across different clinicians as well as institutions, it will be critical to account for all these variables in quantifying the efficacy of integrated BMI-robotic assisted rehabilitation. To examine the current clinical trials underway using these technologies in stroke rehabilitation, we performed a search on the ClinicalTrials.gov registry with the following search terms: "brain computer interface robotic rehabilitation", "robotic-assisted therapy stroke", "brain machine interface stroke". The results of these searches are presented in Table 1. The clinical trials are described here in terms of their primary/secondary outcome measures, in order to survey the outcomes of interest in combined BMI-robotic assisted rehabilitation. Studies including hemiparesis or hemiplegia due to stroke in adults were only considered here. Similarly, only those studies that are still underway are included; terminated studies are excluded. It is important to note that except for two studies, none of the other studies are ready to report results yet. This is indicative of the relatively nascent stages of translation of this research to the clinical settings. Nevertheless, these studies are promising, and we are hopeful that future clinical trials will take into account some of the issues/challenges discussed here while designing their BMI-robotic assisted therapy protocol. For a detailed review of clinical trials involving only robotic rehabilitation, readers are referred to [42•]. In this regard, there is consensus that the field needs guidelines with respect to metrics and regulatory issues for these emergent neurotechnologies. One of the first meetings to address these challenges and discuss potential solutions to accelerate the translation of clinical BMI systems to the end-user was held at The Methodist Hospital Research Institute in February 2013 (http://bmiconference.org/), which brought together about 100 leaders from government, academia, medical centers, industry, foundations, and the patients. A preliminary report [66] reported on key challenges facing the translation of neuroprosthetic technology, including gaps in the scientific data regarding long-term device reliability and safety, uncertainty in the regulatory, market and reimbursement pathways, as well as patient-acceptance challenges that impede fast and effective translation to the end-user. It is clear that a regulatory roadmap and associated guidelines will facilitate innovation and investment in BMI device development, and translation of this neurotechnology to patients in need.

In summary, BMI systems fully integrated with roboticassisted therapy are promising to transform clinical stroke neurorehabilitation. Future research with adaptive, hybrid BMIs can help development of novel clinical outcome 
measures that will not only increase our understanding of neuroplasticity in functional recovery after stroke, but also neuroplasticity after any neurological disease or injury. In the era of personalized medicine with advances in neuromodulation and pharmacogenomics, this neurotechnology has the potential to create innovative personalized rehabilitation medicine, which will maximize functional recovery and reduce the overall healthcare burden caused by physical dysfunction.

Acknowledgments The authors were supported by the National Institute of Neurological Diseases and Stroke (NINDS) Award No. NS081854.

\section{Compliance with Ethics Guidelines}

Conflict of Interest A Venkatakrishnan declares no conflicts of interest. GE Francisco has received research support from the NIH and the TIRR Foundation. JL Contreras-Vidal has received research support from the NIH, NSF, Cullen Foundation and TIRR Foundation.

Human and Animal Rights and Informed Consent This article does not contain any studies with human or animal subjects performed by any of the authors.

\section{References}

Papers of particular interest, published recently, have been highlighted as:

- Of importance

- Of major importance

1. Lloyd-Jones D, Adams RJ, Brown TM, Carnethon M, Dai S, De Simone G, Ferguson TB, Ford E, Furie K, Gillespie C, Go A, Greenlund K, Haase N, Hailpern S, Ho PM, Howard V, Kissela B, Kittner S, Lackland D, Lisabeth L, Marelli A, McDermott MM, Meigs J, Mozaffarian D, Mussolino M, Nichol G, Roger VL, Rosamond W, Sacco R, Sorlie P, Stafford R, Thom T, WasserthielSmoller S, Wong ND, Wylie-Rosett J. Heart disease and stroke statistics-2010 update. Circulation. 2010;121:46-215. doi:10. 1161/CIRCULATIONAHA.109.192667.

2. Velliste M, Perel S, Spalding MC, Whitford AS, Schwartz AB. Cortical control of a prosthetic arm for self-feeding. Nature. 2008;453:1098-101. doi:10.1038/nature06996.

3. McDonald JW, Sadowsky CL, Stampas A. The changing field of rehabilitation: optimizing spontaneous regeneration and functional recovery. Handb Clin Neurol. 2012;109:317-36. doi:10. 1016/B978-0-444-52137-8.00020-6.

4. Dobkin BH. Brain-computer interface technology as a tool to augment plasticity and outcomes for neurological rehabilitation. J Physiol (Lond). 2007;15:489-506.

5. Moreno JC, Ama AJ, Reyes-Guzmán A, Gil-Agudo Á, Ceres R, Pons JL. Neurorobotic and hybrid management of lower limb motor disorders: a review. Med Biol Eng Comput. 2011;49:1119-30. doi10. 1007/s11517-011-0821-4.

6. Rossini PM, Altamura C, Ferreri F, Melgari J-M, Tecchio F, Tombini M, Pasqualetti P, Vernieri F. Neuroimaging experimental studies on brain plasticity in recovery from stroke. Eura Medicophys. 2007;43:241-54.

7. Zollo L, Gallotta E, Guglielmelli E, Sterzi S. Robotic technologies and rehabilitation: new tools for upper-limb therapy and assessment in chronic stroke. Eur J Phys Rehabil Med. 2011;47:223-36.

8. Yozbatiran N, Berliner J, O'Malley MK, Pehlivan AU, Kadivar Z, Boake C, Francisco GE. Robotic training and clinical assessment of upper extremity movements after spinal cord injury: a single case report. J Rehabil Med. 2012;44:186-8. doi:10.2340/ $16501977-0924$.

9. • Aranceta-Garza A, Kumpulainen S, Canela-Repuela M, Boere D, Coronado JL, Egea TG, Francisco GE, Contreras-Vidal JL (2013) Neural Interfaces as Tools for Studying Brain Plasticity. In P. N. Sabesa Biosystems \& Biorobotics. Springer Berlin Heidelberg, Berlin, Heidelberg, pp 89-101. This book chapter presents a case study discussing the potential role of neural interfaces in helping study neural plasticity through changes in cortical dynamics. This is an important step towards closing the knowledge gap in monitoring neural markers of plasticity that can directly impact the design and implementation of rehabilitation therapies.

10. Donchin E, Spencer KM, Wijesinghe R. The mental prosthesis: assessing the speed of a P300-based brain-computer interface. IEEE Trans Rehabil Eng. 2000;8:174-9.

11. Bradberry TJ, Gentili RJ, Contreras-Vidal JL. Reconstructing three-dimensional hand movements from noninvasive electroencephalographic signals. J Neurosci. 2010;30:3432-7. doi:10. 1523/JNEUROSCI.6107-09.2010.

12. Bradberry TJ, Gentili RJ, Contreras-Vidal JL. Fast attainment of computer cursor control with noninvasively acquired brain signals. J Neural Eng. 2011; doi:10.1088/1741-2560/8/3/036010.

13. Buch E, Weber C, Cohen LG, Braun C, Dimyan MA, Ard T, Mellinger J, Caria A, Soekadar S, Fourkas A, Birbaumer N. Think to move: a neuromagnetic brain-computer interface (BCI) system for chronic stroke. Stroke. 2008;39:910-7.

14. Coyle SM, Ward TE, Markham CM. Brain-computer interface using a simplified functional near-infrared spectroscopy system. J Neural Eng. 2007;4:219-26. doi:10.1088/1741-2560/4/3/007.

15. Zimmermann R, Marchal-Crespo L, Edelmann J, Lambercy O, Fluet M-C, Riener R, Wolf M, Gassert R. Detection of motor execution using a hybrid fNIRS-biosignal BCI: a feasibility study. J Neuroeng Rehabil. 2013;10:4. doi:10.1186/1743-0003-10-4.

16. Gentili RJ, Bradberry TJ, Oh H, Hatfield BD, Vidal JLC. Cerebral cortical dynamics during visuomotor transformation: adaptation to a cognitive-motor executive challenge. Psychophysiology. 2011;48:813-24. doi:10.1111/j.1469-8986.2010.01143.x.

17. Gentili RJ, Shewokis PA, Ayaz H, Contreras-Vidal JL. Functional near-infrared spectroscopy-based correlates of prefrontal cortical dynamics during a cognitive-motor executive adaptation task. Front Hum Neurosci. 2013;7:277. doi:10.3389/fnhum.2013.00277.

18. Kilicarslan A, Prasad S, Grossman RG, Contreras-Vidal JL. High accuracy decoding of user intentions using EEG to control a lower-body exoskeleton. Conf Proc IEEE Eng Med Biol Soc. 2013;2013:5606-9. doi:10.1109/EMBC.2013.6610821.

19. Umphred DA, Roller ML, Burton GU, Lazaro RT (2012) Umphred's neurological rehabilitation. Elsevier.

20. Broetz D, Braun C, Weber C, Soekadar SR, Caria A, Birbaumer N. Combination of brain-computer interface training and goal-directed physical therapy in chronic stroke: a case report. Neurorehabil Neural Repair. 2010;24:674-9. doi:10.1177/1545968310368683.

21. • Ramos-Murguialday A, Broetz D, Rea M, Läer L, Yilmaz Ö, Brasil FL, Liberati G, Curado MR, Garcia-Cossio E, GarciaCossio E, Vyziotis A, Cho W, Agostini M, Soares E, Soekadar S, Caria A, Cohen LG. Brain-machine interface in chronic stroke rehabilitation: a controlled study. Ann Neurol. 2013;74:100-8. doi:10.1002/ana.23879. This is one of the first large-scale 
controlled clinical study to demonstrate that stroke patients with minimal hand function who received ERD-driven BMI training as an adjunct to physical therapy to control a hand-orthotic device showed functional improvements in Fugl-Meyer assessment scores, compared to those who received sham BMI training (nonneural control of orthosis).

22. Kaiser V, Kreilinger A, Müller-Putz GR, Neuper C. First steps toward a motor imagery based stroke BCI: new strategy to set up a classifier. Front Neurosci. 2011;5:86. doi:10.3389/fnins.2011. 00086.

23. Sharma N, Cohen LG. Recovery of motor function after stroke. Dev Psychobiol. 2010;52:299-331. doi:10.1002/dev.20508.

24. Takahashi M, Takeda K, Otaka Y, Osu R, Hanakawa T, Gouko $\mathrm{M}$, Ito K. Event related desynchronization-modulated functional electrical stimulation system for stroke rehabilitation: a feasibility study. J Neuroeng Rehabil. 2012;9:56. doi:10.1186/1743-0003-956.

25. Subramanian S, Knaut LA, Beaudoin C, McFadyen BJ, Feldman AG, Levin MF. Virtual reality environments for post-stroke arm rehabilitation. J Neuroeng Rehabil. 2007;4(20):600-2. doi:10. 1186/1743-0003-4-20.

26. Knaut LA, Subramanian SK, McFadyen BJ. Kinematics of pointing movements made in a virtual versus a physical 3-dimensional environment in healthy and stroke subjects. Arch Phys Med Rehabil. 2009;90(5):793-802. doi:10.1016/j.apmr. 2008.10.030.

27. Knaut LA, Magdalon EC, Subramanian S. Virtual reality environments to enhance upper limb functional recovery in patients with hemiparesis. Stud Health Technol Inform. 2009;145:94-108.

28. Bermudez I, Badia S, GarciaMorgade A, Samaha H, Verschure PFMJ. Using a hybrid brain computer interface and virtual reality system to monitor and promote cortical reorganization through motor activity and motor imagery training. IEEE Trans Neural Syst Rehabil Eng. 2013;21:174-81. doi:10.1109/TNSRE.2012. 2229295.

29. Hikosaka O, Nakamura K, Sakai K, Nakahara H. Central mechanisms of motor skill learning. Curr Opin Neurobiol. 2002;12: 217-22.

30. Riener R, Nef T, Colombo G. Robot-aided neurorehabilitation of the upper extremities. Med Biol Eng Comput. 2005;43:2-10. doi:10.1007/BF02345116.

31. Krebs HI, Volpe BT, Williams D, Celestino J, Charles SK, Lynch D, Hogan N. Robot-aided neurorehabilitation: a robot for wrist rehabilitation. IEEE Trans Neural Syst Rehabil Eng. 2007;15:327-35. doi:10.1109/TNSRE.2007.903899.

32. Lo AC, Guarino PD, Richards LG, Haselkorn JK, Wittenberg GF, Federman DG, Ringer RJ, Wagner TH, Krebs HI, Volpe BT, Bever CT Jr, Bravata DM, Duncan PW, Corn BH, Maffucci AD, Nadeau SE, Conroy SS, Powell JM, Huang GD, Peduzzi P. Robot-assisted therapy for long-term upper-limb impairment after stroke. N Engl J Med. 2010;362:1772-83. doi:10.1056/ NEJMoa0911341.

33. Nef T, Mihelj M, Colombo G, Riener R. ARMin-robot for rehabilitation of the upper extremities. IEEE Int Conf Robot Automat. 2006;14:3152-7.

34. Nef T, Mihelj M, Riener R. ARMin: a robot for patient-cooperative arm therapy. Med Biol Eng Comput. 2007;45:887-900. doi:10.1007/s11517-007-0226-6.

35. •• Brokaw EB, Nichols D, Holley RJ, Lum PS. Robotic therapy provides a stimulus for upper limb motor recovery after stroke that is complementary to and distinct from conventional therapy. Neurorehabil Neural Repair. 2013;27(9):816-27. doi:10.1177/ 1545968313510974. This is an interesting preliminary investigation of the use of robotic upper extremity devices in functional training of reach and grasp movements compared to conventional therapy in stroke survivors. The novelty of the findings from this study lie in the fact that they provide clinical evidence for complementary functional benefits of robotic therapy and conventional therapy, which further supports the use of adjunctive robotic therapies in stroke rehabilitation to enhance functional recovery.

36. Vitiello N, Lenzi T, Roccella S, De Rossi SMM, Cattin E, Giovacchini $F$, Vecchi F, Carrozza MC. NEUROExos: a powered elbow exoskeleton for physical rehabilitation. IEEE Trans Robot. 2013;29(1):220-35.

37. Gupta A, O’Malley MK, Patoglu V, Burgar C. Design, control and performance of Ricewrist: a force feedback wrist exoskeleton for rehabilitation and training. Int J Robot Res. 2008;27:233-51. doi:10.1177/0278364907084261.

38. Pehlivan AU, Rose C, O'Malley MK. System characterization of RiceWrist-S: a forearm-wrist exoskeleton for upper extremity rehabilitation. IEEE Int Conf Rehabil Robot. 2013;21:1-6. doi:10.1109/ICORR.2013.6650462.

39. Takahashi CD, Der-Yeghiaian L, Le V, Motiwala RR, Cramer SC. Robot-based hand motor therapy after stroke. Brain. 2008;131:425-37. doi:10.1093/brain/awm311.

40. Loureiro RCV, Harwin WS, Nagai K, Johnson M. Advances in upper limb stroke rehabilitation: a technology push. Med Biol Eng Comput. 2011;49:1103-18. doi:10.1007/s11517-011-0797-0.

41. Yakub F, Md Khudzari AZ, Mori Y. Recent trends for practical rehabilitation robotics, current challenges and the future. Int J Rehabil Res. 2013;36(4):375-8. doi:10.1097/MRR.0000000000000035.

42. - Lo AC. Clinical designs of recent robot rehabilitation trials. Am J Phys Med Rehabil. 2012;91:204-16. doi:10.1097/PHM. $0 \mathrm{~b} 013 \mathrm{e} 31826 \mathrm{bcfa} 3$. This is an important review focusing on the issues in designing and conducting clinical investigations of efficacy of robotic devices. The paper discusses numerous small and large clinical studies involving use of robotic rehabilitation devices, and the various experimental design considerations to maximize effect size and robustness of clinical findings. It is particularly relevant for the potentially large number of clinical studies with robotic devices combined with BMIs in the future. Taking these suggestions into account will help faster translation of these therapeutic technologies from the bench to the clinic while adhering to necessary regulatory standards.

43. Kwakkel G, Kollen BJ, Krebs HI. Effects of robot-assisted therapy on upper limb recovery after stroke: a systematic review. Neurorehabilit Neural Repair. 2008;22:111-21.

44. Jezernik S, Colombo G, Keller T, Frueh H, Morari M. Robotic Orthosis Lokomat ${ }^{\mathrm{TM}}$ : a rehabilitation and research tool. Neuromodulation. 2003;6:108-15. doi:10.1046/j.1525-1403.2003. 03017.x.

45. van der Kooij H, Koopman B, van Asseldonk EHF. Body weight support by virtual model control of an impedance controlled exoskeleton (LOPES ${ }^{\mathrm{TM}}$ ) for gait training. Conf Proc IEEE Eng Med Biol Soc. 2008;2008:1969-72. doi:10.1109/IEMBS.2008. 4649574.

46. Lünenburger L, Colombo G, Riener R, Dietz V. Biofeedback in gait training with the robotic orthosis Lokomat ${ }^{\mathrm{TM}}$. Conf Proc IEEE Eng Med Biol Soc. 2004;7:4888-91. doi:10.1109/IEMBS. 2004.1404352.

47. Veneman JF, Kruidhof R, Hekman EEG, Ekkelenkamp R, van Asseldonk EHF, van der Kooij H. Design and evaluation of the LOPES ${ }^{\mathrm{TM}}$ exoskeleton robot for interactive gait rehabilitation. IEEE Trans Neural Syst Rehabil Eng. 2007;15:379-86. doi:10. 1109/TNSRE.2003.818185.

48. Sawicki GS, Ferris DP. Powered ankle exoskeletons reveal the metabolic cost of plantar flexor mechanical work during walking with longer steps at constant step frequency. J Exp Biol. 2008;212:21-31. doi:10.1242/jeb.017269.

49. Forrester LW, Roy A, Goodman RN, Rietschel J, Barton JE, Krebs HI, Macko RF. Clinical application of a modular ankle robot for 
stroke rehabilitation. Neurorehabilitation. 2013;33:85-97. doi:10. 3233/NRE-130931.

50. Hidler J, Nichols D, Pelliccio M, Brady K, Campbell DD, Kahn JH, Hornby TG. Multicenter randomized clinical trial evaluating the effectiveness of the Lokomat ${ }^{\mathrm{TM}}$ in Subacute Stroke. Neurorehabil Neural Repair. 2008;23:5-13. doi:10.1177/1545968308326632.

51. Schwartz I, Sajin A, Fisher I, Neeb M, Shochina M, Katz-Leurer M, Meiner Z. The effectiveness of locomotor therapy using robotic-assisted gait training in subacute stroke patients: a randomized controlled trial. PM R. 2009;1:516-23. doi:10.1016/j. pmrj.2009.03.009.

52. Koopman B, van Asseldonk EHF, van der Kooij H. Selective control of gait subtasks in robotic gait training: foot clearance support in stroke survivors with a powered exoskeleton. J Neuroeng Rehabil. 2013;10:3. doi:10.1186/1743-0003-10-3.

53. Banala SK, Seok Hun Kim, Agrawal SK, Scholz JP. Robot assisted gait training with active leg exoskeleton (ALEX). IEEE Trans Neural Syst Rehabil Eng. 2009;17:2-8. doi:10.1109/ TNSRE.2008.2008280.

54. Pons JL, Moreno JC, Brunetti FJ, Rocon E. Lower-limb wearable exoskeleton. Rehabilitation Robotics. Vienna: Ed I-Tech Education and Publishing; 2007. p. 471-98.

55. Pons JL. Wearable robots. New York: Wiley; 2008.

56. Bortole M, Pons JL. Development of a exoskeleton for lower limb rehabilitation. In: Moreno JC, editor. Converging clinical and engineering research. Berlin: Springer; 2013. p. 85-90.

57. Kim DH, Shin Y-I, Joa K-L, Shin YK, Lee JJ, You SJH. Immediate effect of Walkbot ${ }^{\mathrm{TM}}$ robotic gait training on neuromechanical knee stiffness in spastic hemiplegia: a case report. Neurorehabilitation. 2013;32:833-8. doi:10.3233/NRE-130907.

58. Pons JL. Rehabilitation exoskeletal robotics. The promise of an emerging field. IEEE Eng Med Biol Mag. 2010;29:57-63. doi:10. 1109/MEMB.2010.936548.

59. Millán JDR, Rupp R, Müller-Putz GR, Murray-Smith R, Giugliemma C, Tangermann M, Vidaurre C, Cincotti F, Kübler A, Leeb R, Neuper C, Müller K-R, Mattia D. Combining braincomputer interfaces and assistive technologies: state-of-the-art and challenges. Front Neurosci. 2010;. doi:10.3389/fnins.2010. 00161 .
60. • Belda-Lois J-M, Mena-del Horno S, Bermejo-Bosch I, Moreno JC, Pons JL, Farina D, Iosa M, Molinari M, Tamburella F, Ramos A, Caria A, Solis-Escalante T, Brunner C, Rea M. Rehabilitation of gait after stroke: a review towards a top-down approach. J Neuroeng Rehabi. 2011;8:66-136. doi:10.1186/1743-0003-866. This is a comprehensive review of the state-of-the-art in lower limb rehabilitation technologies for stroke. The main tenet of the review is that gait rehabilitation strategies can focus on a "top-down" approach, wherein driving neural plasticity can help improve lower extremity function. The paper highlights the fact that systematic investigations of efficacy of lower limb robotic exoskeletons in gait rehabilitation after stroke is lacking, and discusses the therapeutic implications of combining neural interfaces with these devices to improve functional recovery.

61. Daly JJ, Wolpaw JR. Brain-computer interfaces in neurological rehabilitation. Lancet Neurol. 2008;7:1032-43. doi:10.1016/ S1474-4422(08)70223-0.

62. Ang KK, Guan C, Chua KSG, Ang BT, Kuah C, Wang C, Phua KS, Chin ZY, Zhang H. A clinical study of motor imagery-based brain-computer interface for upper limb robotic rehabilitation. Conf Proc IEEE Eng Med Biol Soc. 2009;2009:5981-4. doi:10. 1109/IEMBS.2009.5335381.

63. Ang KK, Guan C, Chua KSG, Ang BT, Kuah C, Wang C, Phua KS, Chin ZY, Zhang H. Clinical study of neurorehabilitation in stroke using EEG-based motor imagery brain-computer interface with robotic feedback. Tornoto: IEEE; 2010. p. 5549-52.

64. Gomez-Rodriguez M, Grosse-Wentrup M, Hill J, Gharabaghi A, Scholkopf B, Peters J. Towards brain-robot interfaces in stroke rehabilitation. IEEE Int Conf Rehabil Robot. 2011;2011:5975385. doi:10.1109/ICORR.2011.5975385.

65. Contreras-Vidal JL, Presacco A, Agashe H, Paek A. Restoration of whole body movement: toward a noninvasive brain-machine interface system. IEEE Pulse. 2012;3:34-7. doi:10.1109/MPUL. 2011.2175635.

66. Liew S-L, Agashe H, Bhagat N, Paek A, Bulea TC. A clinical roadmap for brain-neural machine interfaces: trainees' perspectives on the 2013 international workshop. IEEE Pulse. 2013;4:44-8. doi:10.1109/MPUL.2013.2271686. 